\title{
Analogies for simply supported nonlocal Kirchhoff plates of polygonal planform
}

This paper is dedicated to the memory of Franz Ziegler

Received: 28 March 2017 / Revised: 14 September 2017 / Published online: 22 November 2017

(C) The Author(s) 2017. This article is an open access publication

\begin{abstract}
In the present paper, we consider nonlocal linear elastic Kirchhoff plates, where we restrict to thin, isotropic, and homogeneous plates under the action of static transverse forces, utilizing the differential equation form of the Eringen nonlocal continuum theory. For the case of simply supported plates of polygonal planform, we derive analogies between the solutions of the nonlocal Kirchhoff theory and its local counterpart. First, we extend the Marcus decomposition method for local Kirchhoff plates, where we show that, analogous to the local case considered by Marcus, the moment sum and the nonlocal deflection are both governed by Poisson boundary value problems, which correspond to auxiliary (local) membrane problems. In the present context, it eventually follows that there is a nonvanishing term responsible for a correction of the deflection due to the nonlocal effect, while the moments and shear forces of the local and the nonlocal plate coincide. The nonlocal correction of the deflection turns out to be governed by a membrane-type boundary value problem again. From this fact, it follows immediately that the correction for the deflection due to the nonlocal effect can be derived alternatively from both the local deflection and the local moment sum, which represents a substantial simplification of the nonlocal computations. For the sake of demonstration, we consider examples with closed form solutions. We first consider (as limiting case) infinite plates under the action of single forces, where the singular behavior of Green's function of the nonlocal Kirchhoff theory can be clarified. Then we discuss the bending of nonlocal plate strips for comparison sake, as well as equilateral triangular plates. To our best knowledge, no results for bending of nonlocal triangular plates have been presented in the literature so far. The present paper has been strongly influenced by a former contribution on analogies between simply supported polygonal Kirchhoff plates rigid in shear and shear-deformable plate solutions. This paper was co-authored together with our teacher, the late Professor Franz Ziegler, to whom we dedicate the present paper. In light of this latter contribution, and extending an idea promoted by Professor Franz Ziegler and his co-workers in the framework of various other fields, we finally show in the Appendix that the deflections of simply supported nonlocal Kirchhoff plates can be considered as deflections of a local "background" Kirchhoff plate under the action of additional fictitious eigenstrain loadings, such as thermal moments.
\end{abstract}

Keywords Nonlocal Kirchhoff plates - Simply supported polygonal plates · Membrane-type analogies . Plate-type analogies · Nonlocal triangular plates

\footnotetext{
H. Irschik $(\bowtie)$

Institut für Technische Mechanik, Johannes Kepler University Linz, Linz, Austria

E-mail: hans.irschik@jku.at

URL: www.jku.at/tmech

R. Heuer

Forschungsbereich für Baumechanik und Baudynamik am Institut für Hochbau und Technologie, TU Wien, Vienna, Austria

E-mail: rudolf.heuer@tuwien.ac.at

URL: www.allmech.tuwien.ac.at
} 


\section{Introduction}

The theory of bending of thin linear elastic plates under the action of transverse forces dates back to Sophie Germain, who found the famous fourth-order partial differential equation for the deflection, and to Robert Kirchhoff, who provided a set of consistent boundary conditions for the latter relation, see the monograph by Franz Ziegler on the mechanics of solids and fluids [1] for a comprehensive representation of this so-called Kirchhoff theory of plates rigid in shear. In [1], Ziegler also addressed the decomposition of the fourth-order Germain differential equation for the deflection into two second-order Poisson equations, which involves the moment sum of the plate, in addition to the plate deflection. This decomposition enables a convenient analogy between the plate deflection and the deflection of linear elastic pre-stressed membranes under the action of auxiliary transverse force loadings. This analogy is due to Henri Marcus [2], who suggested using it for deriving particular solutions for plates of general boundary conditions and arbitrary planform. Marcus [2] moreover showed that this analogy is complete for simply supported plates of polygonal planform.

In extension of the original Kirchhoff theory, various shear-deformable plate theories for thick plates, as well as theories for nonisotropic, nonhomogeneous, and nonelastic plates were eventually derived in the literature. In the last decades, plate theories were also extended in order to include certain nonlocal effects. An important class of nonlocal plate theories emerges from the celebrated linear theory of nonlocal continuum mechanics by Ahmed Cemal Eringen, see [3]. In this theory, the stress at some reference point is described as a functional of the strain field at every point in the body, which leads to a set of integro-partial differential equations. By means of these integral constitutive relations and their kernel function, which characterizes the nonlocal behavior of the continuum, an additional length parameter is eventually involved, which is intended to reflect the effect of long-range inter-atomic forces, and which thus becomes particularly significant in micro- or nanoscale structures. However, the integral continuum relations are generally difficult to solve. Now, exploiting that the kernel in the integral formulation represents a Green's function of a linear differential operator, it has been shown by Eringen [4] that the integral constitutive relations can be transformed into an equivalent set of (singular) partial differential equations, which provides considerable simplicity over the original integral equations. For the sake of convenience, this differential form of the Eringen nonlocal continuum theory has been often utilized for structural applications, where a comparatively large corpus of papers on nonlocal structures, such as beams and plates, has emerged within a relatively short period of time in the literature. For fundamental contributions to the Eringen differential equation-type nonlocal theory of plates, see, e.g., Lu et. al. [5] and Reddy [6], where not only isotropic linear elastic Kirchhoff plates rigid in shear have been treated, but also nonisotropic, nonlinear, and shear-deformable plates were considered.

It has been noted, however, in [4] that the above-mentioned Green-type kernels, which allow a transformation of the original integral formulations to the differential ones, represent approximations only, being valid when the reference point, for which the constitutive relations are formulated, is not too close to the boundaries of the body.

It thus appears to be not surprising that certain problems and paradoxical results associated with the proper formulation of boundary conditions have increasingly been discussed in the literature on nonlocal structures in the last years. For instance, see [7] ad [8] for comments and discussions on the proper extension of the Navier series representation to nonlocal rectangular plates when the transverse loading does not vanish at the boundary. For beams, paradoxical results have been particularly encountered when clamped boundaries are involved, such as in the case of cantilevers, where an apparent stiffening effect for distributed loads was obtained in contrast to other boundary conditions for which a softening effect can be observed. Recent suggestions for a resolution of such paradoxes in beams appear to be far reaching, ranging from a possible nonequivalence of the outcomes of the nonlocal integral and differential formulations, see [9], to a combination of the nonlocal differential model with certain additional discontinuity requirements, see [10], or to the study of the possible nonexistence of solutions in certain cases, see [11].

So far, these controversial discussions have not yet been extended from the one-dimensional case of bending of beams to two-dimensional plate bending problems. On the other hand, a literature survey shows that some classes of more complex plate geometries have not even found a general treatment in the literature on nonlocal continua so far, such as the practically important case of polygonal plates. In the present contribution, we thus deal with such geometries, where we restrict to the nonlocal Kirchhoff theory of thin, isotropic, and homogeneous linear elastic plates rigid in shear, since the latter framework represents a topic in its own right, also at the micro- or nanoscale. In light of the above-mentioned controversial discussions, we particularly consider plates that are simply supported all around the boundary, since results for the corresponding beamtype counterpart, the simply supported one-span beam, have found consensus in the literature. When there 
was no consensus reported, such as in [11], the discrepancies between different formulations have turned out to be small. Hence, in the following we deal with simply supported nonlocal Kirchhoff plates of arbitrary polygonal planform under static transverse force loading in the framework of the Eringen differential nonlocal formulation.

Subsequently, we intend to solve the nonlocal Kirchhoff plate bending problems under consideration by applying the Marcus local decomposition method [2] by analogy. Following this hypothesis, we subsequently manage to find particular solutions of the Eringen nonlocal differential plate theory for deflections, bending moments, and shear forces in terms of the deflections of auxiliary (local) membrane problems. In case of simply supported straight edges, by adding proper homogeneous boundary conditions to the auxiliary membrane problems, we find that the corresponding set of particular solutions satisfies both the differential equations and the boundary conditions of the Eringen nonlocal plate problem. Supposing uniqueness, we thus arrive at a general procedure for deriving the complete solutions of simply supported nonlocal plates of arbitrary polygonal planform by using auxiliary membrane problems; that is, the proposed Marcus-type analogy turns out to be complete in the nonlocal case. This finding holds also for infinitely extended plates. We exemplarily will show this subsequently for the Green's functions of the infinite plate, where we clarify the singular behavior of nonlocal plates under the action of single forces. For convenience, we decompose the nonlocal deflection and the nonlocal stress resultants further into their local counterparts and into additional corrections due to the nonlocal effect. The local parts correspond to the classical local Kirchhoff plate theory, as represented by the Marcus decomposition method [2]. It is found that the correction for the deflection represents a softening behavior, as this is to be expected. It follows immediately that the correction for the deflection is proportional to both the local moment sum and the Laplace derivative of the local deflection. The correction terms for the stress resultants moreover turn out to vanish in this case of simply supported polygonal plates. As illustrative special examples, we concentrate on problems with closed form solutions and present results for an infinite plate under the action of a single force, a uniformly loaded plate strip, as well as for an uniformly loaded equilateral triangular plate. The results for the plate strip correspond to results from the literature for the similar problem of nonlocal simply supported one-span beams. To our best knowledge, no results have been presented in the literature so far for nonlocal equilateral triangular plates. In the framework of our solution strategy, the nonlocal results can be completely obtained from results derived originally by Woinowsky-Krieger [12] for classical local membranes and Kirchhoff plates of triangular planform.

Our subsequent derivations are strongly motivated by some strategies addressed in [13], a paper which the present authors have co-authored together with their academic teacher, the late Professor Franz Ziegler, to whom we devote our present contribution. In [13], among a discussion of other works, a review was presented on previous contributions of the group of Professor Ziegler to static and dynamic analogies between local Kirchhoff plates, membranes, and shear-deformable plates. Several shear-deformable plate formulations and various complicating effects were addressed in [13]; for a study on some eigenvalue problems of simply supported polygonal membranes and plates, see, e.g., [14]. The series of papers discussed in [13] started with a static study presented in [15], in which it was shown that a complete analogy exists between infinite and simply supported polygonal Kirchhoff plates and some of their shear-deformable counterparts, where an auxiliary thermal loading of the Kirchhoff plates was introduced. It was moreover shown in [15] that for certain shear-deformable theories the stress resultants are not affected by the influence of shear, similar to our below-derived results on the stress resultants of nonlocal Kirchhoff plates. A main idea of [13] was to describe the effect of shear as an auxiliary eigenstrain loading, such as a thermal loading in a Kirchhoff background plate rigid in shear. This idea was promoted in a generalized form by Professor Franz Ziegler and his co-authors in various other fields, such as for nonlinear structural mechanics and dynamic viscoplasticity, see [16] for a plate-type application, and [16] and [17] for reviews. Motivated by this background strategy, we subsequently also show that simply supported polygonal nonlocal Kirchhoff plates can be treated as local background Kirchhoff plates with an additional eigenstrain loading, see the Appendix of the present manuscript.

\section{Fundamental relations of the Eringen nonlocal differential theory of Kirchhoff plates}

The following notation is used for stress resultants in the nonlocal plate: Bending and twisting moments are written as $M_{x x}, M_{y y}$, and $M_{x y}=M_{y x}$, respectively, and shear forces are denoted by $Q_{x}$ and $Q_{y}$. A Cartesian coordinate system is used, where the $(x, y)$ coordinates are situated in the mid-surface of the homogeneous plate with constant thickness $h$. Considering the equilibrium equations for these stress resultants, 


$$
\begin{aligned}
& Q_{x}=\frac{\partial M_{x x}}{\partial x}+\frac{\partial M_{x y}}{\partial y}, \\
& Q_{y}=\frac{\partial M_{x y}}{\partial x}+\frac{\partial M_{y y}}{\partial y}, \\
& \frac{\partial Q_{x}}{\partial x}+\frac{\partial Q_{y}}{\partial y}=-q,
\end{aligned}
$$

elimination of the shear forces leads to

$$
\frac{\partial^{2} M_{x x}}{\partial x^{2}}+2 \frac{\partial^{2} M_{x y}}{\partial x \partial y}+\frac{\partial^{2} M_{y y}}{\partial y^{2}}=-q .
$$

The transverse force loading per unit area of the plate is denoted as $q$. Assuming small deformations of the plate, the influence of in-plane forces, if any, has been neglected in Eq. (1.3), see Reddy [6] for geometrically nonlinear extensions. Note that the equilibrium relations of the local and the nonlocal plate theory coincide, compare, e.g., Ziegler [1] and Reddy [6], similar to the fact that these relations hold for both the Kirchhoff theory of thin plates rigid in shear and its shear-deformable extensions that date back to Eric Reissner and Raymond D. Mindlin, see the discussion given in [13].

Within the scope of the Eringen nonlocal differential formulation, the corresponding constitutive relations of a homogeneous, isotropic, and linear elastic Kirchhoff plate turn out to be

$$
\begin{aligned}
& \left(1-\lambda \nabla^{2}\right) M_{x x}=-D\left(\frac{\partial^{2} w}{\partial x^{2}}+v \frac{\partial^{2} w}{\partial y^{2}}\right), \\
& \left(1-\lambda \nabla^{2}\right) M_{y y}=-D\left(\frac{\partial^{2} w}{\partial y^{2}}+v \frac{\partial^{2} w}{\partial x^{2}}\right), \\
& \left(1-\lambda \nabla^{2}\right) M_{x y}=-D(1-v) \frac{\partial^{2} w}{\partial x \partial y}
\end{aligned}
$$

where $w$ denotes the transverse deflection of the nonlocal isotropic elastic Kirchhoff plate, see, e.g., [5] and [6]. Nonhomogeneous, anisotropic, and nonlinear effects have been treated in the latter papers also. In Eq. (3), the bending stiffness of the plate with constant thickness $h$ is $D=E h^{3} / 12\left(1-v^{2}\right)$, where $E$ denotes Young's modulus and $v$ stands for Poisson's ratio. The two-dimensional Laplace operator is $\nabla^{2}=\partial^{2} / \partial x^{2}+\partial^{2} / \partial y^{2}$. The nonlocal parameter in Eq. (3) is given by $\lambda=\left(a e_{0}\right)^{2} \geq 0$. Here, $a$ stands for an internal characteristic length, e.g., a lattice parameter, a granular size or a molecular diameter, brought in by the specific nonlocal context under consideration, and $e_{0}$ is a respective material constant, see [3-6]. For $\lambda=0$, the classical local constitutive plate relations, see Ziegler [1], are obtained. In any case, Eqs. (1) and (3) form a set of six partial differential equations for the three moments, the two shear forces and the deflection of the plate.

Applying the operator $\left(1-\lambda \nabla^{2}\right)$ to Eq. (2), and using the interchangeability of differentiation and Eq. (3), gives the governing differential equation for the deflection of a nonlocal Kirchhoff plate,

$$
D\left(\frac{\partial^{4} w}{\partial x^{4}}+2 \frac{\partial^{4} w}{\partial x^{2} \partial y^{2}}+\frac{\partial^{4} w}{\partial y^{4}}\right)=D \nabla^{2} \nabla^{2} w=\left(1-\lambda \nabla^{2}\right) q .
$$

In the classical local plate theory, i.e., for $\lambda=0$, Eqs. (1) and (3) can be used in order to express moments and shear forces directly by higher-order derivatives of the deflection. Hence, in the local theory, using Eq. (4) as the governing differential equation, complete boundary value problems for the deflection can be easily set up also when moments or shear forces are prescribed at the boundary of the plate, see, e.g., [1]. In the nonlocal theory, however, for $\lambda>0$, this procedure is not straightforward, due to the Laplace operator appearing at the left-hand sides of Eq. (3). To the understanding of the present authors, this fact constitutes the mathematical background of the controversial discussions in the literature that we have mentioned above in the Introduction. Moreover, the effect of the term $\nabla^{2} q$ on the right-hand side of Eq. (4) needs special consideration. Note, however, that analogous loading terms appear for shear-deformable local plates, or for local plates with an eigenstrain loading, such as a thermal loading, see [13]. These classical solutions can serve as guidelines.

For a resolution, we now study the effect of introducing the Marcus moment sum [2] in the above nonlocal relations. With the moment sum

$$
M=\frac{M_{x x}+M_{y y}}{1+v}
$$


one gets from Eq. (3):

$$
D \nabla^{2} w=-\left(1-\lambda \nabla^{2}\right) M
$$

and further, by means of Eq. (4), we find that

$$
\left(1-\lambda \nabla^{2}\right)\left(\nabla^{2} M+q\right)=0 .
$$

Applying the operator $\nabla^{2}$ onto Eq. (3) and taking into account Eq. (6), it follows that

$$
\begin{aligned}
& \left(1-\lambda \nabla^{2}\right)\left(\nabla^{2} M_{x x}-\frac{\partial^{2} M}{\partial x^{2}}-v \frac{\partial^{2} M}{\partial y^{2}}\right)=0, \\
& \left(1-\lambda \nabla^{2}\right)\left(\nabla^{2} M_{y y}-\frac{\partial^{2} M}{\partial y^{2}}-v \frac{\partial^{2} M}{\partial x^{2}}\right)=0, \\
& \left(1-\lambda \nabla^{2}\right)\left(\nabla^{2} M_{x y}-(1-v) \frac{\partial^{2} M}{\partial x \partial y}\right)=0 .
\end{aligned}
$$

Note that in the local limit, for $\lambda=0$, Eqs. (6)-(8) correspond to results that date back to Marcus [2], see also Ziegler [1] for Eqs. (6) and (7). After discussing simply supported boundary conditions in more detail, relations (5)-(8) will be used to obtain particular and eventually complete solutions for nonlocal plate problems.

\section{Boundary conditions at straight, simply supported edges of nonlocal Kirchhoff plates}

In the literature on nonlocal plates, there is consensus that the deflection and the edge bending moment must be prescribed at a simply supported edge $\Gamma$ of a Kirchhoff plate, see [5] and [6], in the same manner, as this is the case in the local version of the Kirchhoff theory, see [1] and [2]. We consider straight edges $\Gamma$ assigned with local $(n, s)$ Cartesian coordinate systems, where $n$ denotes the outer normal coordinate, and $s$ points along the edge, and we consider homogeneous boundary conditions. Hence, for polygonal plates of simply supported boundaries, the boundary conditions can be written as

$$
\Gamma: \quad w=0, \quad M_{n n}=0 .
$$

The question arises how to relate the second boundary condition in Eq. (9.1), the condition of a vanishing bending moment, to the deflection $w$ and its derivatives, in order to have a complete boundary value problem for the fourth-order differential equation for the deflection stated in Eq. (4). In light of the terms containing $\lambda$ in Eq. (3), this question cannot be answered in a straightforward manner. Since we consider straight edges, the first boundary condition in Eq. (9.1) for the deflection yields, see Eq. (6):

$$
\Gamma: \quad \frac{\partial w}{\partial s}=0, \quad \frac{\partial^{2} w}{\partial s^{2}}=0, \quad \Rightarrow \quad D \nabla^{2} w=D \frac{\partial^{2} w}{\partial n^{2}}=-\left(1-\lambda \nabla^{2}\right) M .
$$

Now, in order to suggest a suitable boundary condition in $w$, which might replace the bending moment boundary condition in Eq. (9.1), we here assume that $\nabla^{2} M+q=0$. This latter relation, indeed, represents a particular solution of Eq. (7). Note that the completeness of this solution in the case of simply supported polygonal nonlocal plates will be established later on, in Sec. 4 below. With this suggestion, Eq. (9.2) becomes

$$
\Gamma: \quad D \frac{\partial^{2} w}{\partial n^{2}}=-(M+\lambda q)
$$

Using Eq. (3), it follows eventually from the bending moment boundary condition in Eq. (9.1) that

$$
\Gamma:\left(1-\lambda \nabla^{2}\right) M_{n n}=-\lambda \nabla^{2} M_{n n}=-D \frac{\partial^{2} w}{\partial n^{2}}=M+\lambda q .
$$

Note that in this latter relation neither $M$ nor $\nabla^{2} M_{n n}$ are known in advance at $\Gamma$; particularly, $\nabla^{2} M_{n n}$ generally will not vanish at $\Gamma$. 
Now, let us assume moreover that there is

$$
\Gamma: \quad M=0,
$$

as this does hold in the classical local Kirchhoff theory at straight simply supported edges, see [1] and [2]. This latter assumption immediately leads to $M_{s s}=0$ at $\Gamma$, see Eq. (5). Moreover, the following boundary condition in terms of the deflection then follows from Eq. (9.3), replacing the second condition in Eq. (9.1):

$$
\Gamma: \quad D \frac{\partial^{2} w}{\partial n^{2}}=-\lambda q .
$$

An analogous boundary condition is well accepted in the literature on nonlocal simply supported beams, see [8]. Using Eq. (10), we obtain from Eq. (3):

$$
-\lambda \nabla^{2} M_{n n}=-D \frac{\partial^{2} w}{\partial n^{2}}=\lambda q, \quad-\lambda \nabla^{2} M_{s s}=-D \nu \frac{\partial^{2} w}{\partial n^{2}}=\nu \lambda q .
$$

Note that, from a mathematical point of view, Eq. (11) can be used as a suitable boundary condition for the differential equation Eq. (4), additional to the deflection boundary condition in Eq. (9.1). In the context of the nonlocal plate theory, the physical appropriateness of Eq. (11), however, must be yet established, which will be undertaken subsequently, using the Marcus decomposition method [2] by analogy.

\section{Particular and complete solutions of the Eringen nonlocal differential theory of Kirchhoff plates}

Our subsequent procedure is the following: We start with a set of particular solutions of the relations presented in Sect. 2 above by skipping the common operator $\left(1-\lambda \nabla^{2}\right)$ in Eqs. (7) and (8). Then we assign the boundary condition (10) to the resulting relation for the moment sum. This gives a Poisson boundary value problem for the moment sum, which is analogous to the one for the deflection of a pre-stressed auxiliary membrane, see [1] and [2]. The resulting moment sum is used in Eq. (6), which, together with the deflection boundary condition in Eq. (9.1), forms a Poisson boundary value problem for the deflection that again is analogous to the deflection of a pre-stressed membrane. The particular solution for the moment sum is also used in Eq. (8), in order to obtain particular solutions for the moments. The particular solutions so derived satisfy the nonlocal differential relations presented in Sect. 2. However, they also satisfy the boundary conditions for a nonlocal Kirchhoff plate with simply supported straight edges presented in Eq. (9.1). This becomes clear by splitting each entity into its local part, which does not contain the factor $\lambda$, and the additional nonlocal correction term due to the nonlocal parameter $\lambda$. Hence, our procedure leads to complete solutions for simply supported nonlocal Kirchhoff plates of arbitrary polygonal planform. In more detail, the procedure is as follows:

A particular solution of Eq. (7) can be found as

$$
\begin{aligned}
\nabla^{2} M & =-q, \\
\Gamma: M & =0 .
\end{aligned}
$$

This Poisson boundary value problem is of the same form as the one for a pre-stressed, transversely loaded membrane, but it also holds for simply supported polygonal Kirchhoff plates according to the local theory, see [1] and [2]. The solution of this boundary value problem is either known from the literature or can be accessed by well-known methods, see [1] and [2]. We now decompose $M$ into its local part and the nonlocal correction term:

$$
\begin{aligned}
& M=M_{l}+M_{\lambda}, \\
& \nabla^{2}\left(M_{l}+M_{\lambda}\right)=-q, \\
& \Gamma: \quad M_{l}+M_{\lambda}=0 .
\end{aligned}
$$

We thus arrive at the following two auxiliary membrane problems:

$$
\begin{aligned}
& \nabla^{2} M_{l}=-q, \\
& \Gamma: \quad M_{l}=0,
\end{aligned}
$$


and

$$
\begin{aligned}
& \nabla^{2} M_{\lambda}=0, \\
& \Gamma: \quad M_{\lambda}=0 .
\end{aligned}
$$

Equations (16) and (17) are satisfied if

$$
M=M_{l}, \quad M_{\lambda}=0 .
$$

Hence, the particular moment sum $M$ is not affected by the nonlocal parameter $\lambda$, as this is immediately clear from Eqs. (13) and (14). Rather, the moment sum is equal to the corresponding classical local one. Substituting this result into Eq. (6) and using Eq. (9.1), we again obtain a Poisson boundary value problem, which is of the form of that for a pre-stressed, transversely loaded membrane, the auxiliary transverse loading this time being formed by $M_{l}+\lambda q$ :

$$
\begin{aligned}
D \nabla^{2} w & =-(M+\lambda q)=-\left(M_{l}+\lambda q\right), \\
\Gamma: \quad w & =0 .
\end{aligned}
$$

Since the first term inside the bracket at the right-hand side of Eq. (19.1), $M_{l}$, is not affected by the nonlocal parameter, a decomposition of the deflection into the classical local deflection $w_{l}$ and the correction term $w_{\lambda}$ due to the nonlocal parameter $\lambda$ makes sense:

$$
w=w_{l}+w_{\lambda} .
$$

This results in the following two individual boundary value problems of the Poisson type for the classical local deflection and the correction term due to the nonlocal parameter $\lambda$ :

$$
\begin{aligned}
D \nabla^{2} w_{l} & =-M_{l}, \\
\Gamma: \quad w_{l} & =0,
\end{aligned}
$$

and

$$
\begin{aligned}
D \nabla^{2} w_{\lambda} & =-\lambda q, \\
\Gamma: w_{\lambda} & =0 .
\end{aligned}
$$

The first problem, Eqs. (21), reflects a well-known analogy for deflection of classical local Kirchhoff plates, see [1] and [2]. The second problem, Eqs. (22), is of the form of both Eqs. (21) and (16). This allows computing the nonlocal correction for the deflection, $w_{\lambda}$, from both, the moment sum and the deflection of the classical local Kirchoff plate: Comparing Eq. (22) with Eqs. (16) and (21) it turns out that

$$
w_{\lambda}=\frac{\lambda M_{l}}{D}=-\lambda \nabla^{2} w_{l}
$$

Note that adding Eqs. (21.1) and (22.1), and applying the result to the straight boundary $\Gamma$ by using Eqs. (16.2), (21.2) and (22.2), indeed leads to the boundary condition Eq. (11), which has been postulated in the previous Sect. 3.

Particular solutions for the Laplace derivatives of the nonlocal bending and twisting moments follow from Eqs. (8) by skipping the common operator $\left(1-\lambda \nabla^{2}\right)$ and substituting Eq. (18):

$$
\begin{aligned}
& \nabla^{2} M_{x x}=\frac{\partial^{2} M}{\partial x^{2}}+v \frac{\partial^{2} M}{\partial y^{2}}=\frac{\partial^{2} M_{l}}{\partial x^{2}}+v \frac{\partial^{2} M_{l}}{\partial y^{2}}, \\
& \nabla^{2} M_{y y}=\frac{\partial^{2} M}{\partial y^{2}}+v \frac{\partial^{2} M}{\partial x^{2}}=\frac{\partial^{2} M_{l}}{\partial y^{2}}+v \frac{\partial^{2} M_{l}}{\partial x^{2}}, \\
& \nabla^{2} M_{x y}=(1-v) \frac{\partial^{2} M}{\partial x \partial y}=(1-v) \frac{\partial^{2} M_{l}}{\partial x \partial y} .
\end{aligned}
$$


Analogous to the moment sum, see Eq. (15.1), and to the deflection, see Eq. (20), the bending moments now are decomposed into their classical local portions and the correction term due to the nonlocal parameter $\lambda$ :

$$
\begin{aligned}
& M_{x x}=M_{x x l}+M_{x x \lambda}, \\
& M_{y y}=M_{y y l}+M_{y y \lambda}, \\
& M_{x y}=M_{x y l}+M_{x y \lambda} .
\end{aligned}
$$

The constitutive relations for the classical local Kirchhoff theory read, see [1] and [2]:

$$
\begin{aligned}
& M_{x x l}=-D\left(\frac{\partial^{2} w_{l}}{\partial x^{2}}+v \frac{\partial^{2} w_{l}}{\partial y^{2}}\right), \\
& M_{y y l}=-D\left(\frac{\partial^{2} w_{l}}{\partial y^{2}}+v \frac{\partial^{2} w_{l}}{\partial x^{2}}\right), \\
& M_{x y l}=-D(1-v) \frac{\partial^{2} w_{l}}{\partial x \partial y} .
\end{aligned}
$$

Substituting Eqs. (25) and (26) into the nonlocal constitutive relations, Eq. (3), and using the decomposition in Eq. (20) for the deflection, gives the following expressions for the correction terms:

$$
\begin{aligned}
& M_{x x \lambda}=-D\left(\frac{\partial^{2} w_{\lambda}}{\partial x^{2}}+v \frac{\partial^{2} w_{\lambda}}{\partial y^{2}}\right)+\lambda \nabla^{2} M_{x x}, \\
& M_{y y \lambda}=-D\left(\frac{\partial^{2} w_{\lambda}}{\partial y^{2}}+v \frac{\partial^{2} w_{\lambda}}{\partial x^{2}}\right)+\lambda \nabla^{2} M_{y y}, \\
& M_{x y \lambda}=-D(1-v) \frac{\partial^{2} w_{\lambda}}{\partial x \partial y}+\lambda \nabla^{2} M_{x y} .
\end{aligned}
$$

Now, substituting into the latter relations the results of Eq. (24), and considering Eq. (23), it is found that these correction terms do vanish:

$$
\begin{array}{ll}
M_{x x \lambda}=0, & M_{x x}=M_{x x l}, \\
M_{y y \lambda}=0, & M_{y y}=M_{y y l}, \\
M_{x y \lambda}=0, & M_{x y}=M_{x y l} .
\end{array}
$$

We thus have obtained the following set of particular solutions of the Eringen nonlocal Kirchhoff problem: The local classical solution for the deflection $w_{l}$ is corrected by $w_{\lambda}$ as stated in Eq. (20). The deflection correction $w_{\lambda}$ satisfies a Poisson boundary value problem that is analogous to the deflection of a pre-stressed membrane, Eq. (22). The correction terms for the moments do vanish, Eq. (28), such that the nonlocal moments coincide with their classical local counterparts. But $w_{l}, w_{\lambda}$ as well as the local bending moments satisfy the boundary conditions for polygonal plates with simply supported edges that were stated in Eq. (9.1). Hence, we conclude that our set of particular solutions represents the complete solution set for this class of nonlocal Kirchhoff plates. This is exemplified in the next Section.

\section{Example problems}

\subsection{Green's function of the infinite nonlocal plate}

As our first example, we consider the infinitely extended nonlocal plate. The fundamental behavior of infinite plates under the action of a single force $F$ is commonly used in order to describe the singular behavior of plates near the application point of single forces in plates of finite extension also, see, e.g., [12] for local triangular plates. Solutions due to single forces or moments are called Green's functions of the plate problem under consideration, see, e.g., [19], where solutions for the infinite plate have been used as singular parts of Green's functions for rectangular plates in the context of a boundary element method. In a polar $(r, \varphi)$ coordinate system 
with origin located at the point of application of $F$, the Green's function for the deflection of the infinite plate according to the local Kirchhoff theory reads, see, e.g., [1] and [19]:

$$
w_{l \infty}^{F}=\frac{F r^{2}}{8 \pi D} \ln \frac{r}{b} \text {. }
$$

From this fundamental solution, which is regular at $r \rightarrow 0$, the corresponding local moment sum in the infinite local Kirchhoff plate becomes singular at the application point, see [19]:

$$
M_{l \infty}^{F}=-D \nabla^{2} w_{l \infty}^{F}=-D\left(\frac{\mathrm{d}^{2}}{\mathrm{~d} r^{2}}+\frac{1}{r} \frac{\mathrm{d}}{\mathrm{d} r}\right) w_{l \infty}^{F}=-\frac{F}{2 \pi}\left(\ln \frac{r}{b}+1\right) .
$$

Following Eq. (23), the nonlocal correction term for the deflection becomes

$$
w_{\lambda \infty}^{F}=\frac{\lambda M_{l \infty}^{F}}{D}=-\frac{\lambda F}{2 \pi D}\left(\ln \frac{r}{b}+1\right) .
$$

It is thus seen that the Eringen differential theory of nonlocal Kirchhoff plates delivers a logarithmic singularity of the deflection due to a single force. The singular behavior of the nonlocal stress resultants, however, is identical to that of the local Kirchhoff theory.

\subsection{Infinite nonlocal plate strip with simply supported edges}

Next, we consider a plate strip of span $a$, the strip being infinitely extended in the direction of the $y$ coordinate. The $x$ coordinate becomes perpendicular to the edges of the strip, $n \triangleq x$, where we choose the origin of the coordinate system at one edge, such that $0 \leq x \leq a$. We take the edges of the strip to be simply supported. The boundary conditions in Eq. (9.1) now read

$$
\Gamma(\triangleq x=0, a): \quad w=0, \quad M_{x x}=0 .
$$

We now assume that the loading of the plate varies with $x$ only, $q=q(x)$. Consequently the strip is bent to a cylindrical surface. It then turns out, see Ziegler [1], that the local displacement, $w_{l}$, and the local bending moment, $M_{x x l}$, of the Kirchhoff plate strip correspond to the well-known analogous solutions for displacement and bending moment, respectively, of a simply supported one-span local Bernoulli-Euler beam, where the bending stiffness of the beam is to be replaced by the plate stiffness $D$. Moreover, in the local plate there is

$$
M_{y y l}=v M_{x x l}, \quad M_{l}=M_{x x l} .
$$

At first, a strip with constant loading is considered, $q=q_{0}=$ const. The local displacement becomes, see Ziegler [1]:

$$
w_{l}=\frac{q_{0} a^{4}}{D}\left(\frac{1}{24}\left(\frac{x}{a}\right)^{4}-\frac{1}{12}\left(\frac{x}{a}\right)^{3}+\frac{1}{24} \frac{x}{a}\right) .
$$

The local bending moments are

$$
M_{x x l}=M_{l}=\frac{q_{0} a^{2}}{2}\left(\frac{x}{a}-\left(\frac{x}{a}\right)^{2}\right), \quad M_{y y l}=v M_{x x l}, \quad M_{x y l}=0 .
$$

The correction for the nonlocal deflection is obtained from Eq. (23) as

$$
w_{\lambda}=\lambda \frac{q_{0} a^{2}}{2 D}\left(\frac{x}{a}-\left(\frac{x}{a}\right)^{2}\right), \quad w_{\lambda}\left(\frac{a}{2}\right)=\lambda \frac{q_{0} a^{2}}{8 D} .
$$

This corresponds to a result presented before for nonlocal beams, e.g., by Challamel and Reddy [8], demonstrating the softening effect of the nonlocal parameter $\lambda$. The nonlocal correction terms for the moments vanish, as this is obvious in the present statically determinate case. It can be easily checked that the governing differential equations of the Eringen nonlocal theory, Eqs. (1) and (3), and the boundary conditions, Eq. (32), are indeed satisfied by Eqs. (34)-(36). 
As a second loading case, a constant line load is applied at the mid-span of the strip, $q=p_{0} \delta(x-a / 2)$, where $\delta$ stands for the Dirac delta function. Here we restrict only to the nonlocal correction for the deflection. Following the above-mentioned beam analogy, the bending moment of the strip is obtained from beam equilibrium considerations, see, e.g., Ziegler [1]:

$$
0 \leq x \leq \frac{a}{2}: \quad M_{x x l}=M_{l}=\frac{p_{0}}{2} x
$$

From Eq. (23), the correction term for the nonlocal deflection is thus obtained as

$$
w_{\lambda}=\frac{\lambda M_{l}}{D}=\lambda \frac{p_{0}}{2 D} x, \quad w_{\lambda}\left(\frac{a}{2}\right)=\lambda \frac{p_{0} a}{4 D} .
$$

This is a result which has been stated for nonlocal beams before by Wang and Liew [20]. Our present explanation for the fact that the correction for the deflection due to the nonlocal parameter $\lambda$ shows a kink at the point of application of the line load is that $w_{\lambda}$ must be proportional to the moment in Eq. (37). Note that this nonsmooth behavior is analogous to the singular behavior at the point of application of a single force, as discussed above, see Eq. (31).

\subsection{Nonlocal equilateral triangular plate with simply supported edges}

We consider an equilateral triangular plate, where the height of the triangle is denoted as $a$. The origin of the coordinate system is located at the centroid of the triangle, where the $x$-axis is taken as perpendicular to one edge, pointing toward the corner opposite to this edge, see Woinowsky-Krieger [12]. Assuming a constant load, $q=q_{0}=$ const., the moment sum and the deflection of the plate according to the local Kirchhoff theory are, see [12]:

$$
\begin{aligned}
& M_{l}=\frac{q_{0}}{4 a}\left[x^{3}-3 y^{2} x-a\left(x^{2}+y^{2}\right)+\frac{4}{27} a^{3}\right], \\
& w_{l}=\frac{q_{0}}{64 a D}\left[x^{3}-3 y^{2} x-a\left(x^{2}+y^{2}\right)+\frac{4}{27} a^{3}\right]\left(\frac{4}{9} a^{2}-x^{2}-y^{2}\right) .
\end{aligned}
$$

Using Eq. (23), the correction of the deflection due to the nonlocal effect thus becomes:

$$
w_{\lambda}=\frac{\lambda M_{l}}{D}=-\lambda \nabla^{2} w_{l}=\frac{\lambda q_{0}}{4 a D}\left[x^{3}-3 y^{2} x-a\left(x^{2}+y^{2}\right)+\frac{4}{27} a^{3}\right] .
$$

Hence, the nonlocal moment sum and the nonlocal bending and twisting moments turn out to be equal to their local counterparts, see Eqs. (18) and (28). In order to give evidence for this, we compute the Laplace operators of the moments, using Eq. (40) and the local constitutive relations, Eq. (26):

$$
\begin{aligned}
\nabla^{2} M_{x x} & =\nabla^{2} M_{x x l}=\frac{q_{0}}{4 a}[6 x-2 a-v(6 x+2 a)], \\
\nabla^{2} M_{y y} & =\nabla^{2} M_{y y l}=\frac{q_{0}}{4 a}[-6 x-2 a+v(6 x-2 a)], \\
\nabla^{2} M_{x y} & =\nabla^{2} M_{x y l}=-\frac{3}{2} \frac{q_{0}}{a}(1-v) y .
\end{aligned}
$$

Substituting this together with the nonlocal correction of the deflection, Eq. (41), into Eq. (27), it is found that the correction terms for the moments due to the nonlocal effect do vanish also in the case under consideration:

$$
\begin{aligned}
M_{x x \lambda} & =-D\left(\frac{\partial^{2} w_{\lambda}}{\partial x^{2}}+v \frac{\partial^{2} w_{\lambda}}{\partial y^{2}}\right)+\lambda \nabla^{2} M_{x x} \\
& =-\frac{\lambda q_{0}}{4 a}[6 x-2 a-v(6 x+2 a)]+\frac{\lambda q_{0}}{4 a}[6 x-2 a-v(6 x+2 a)]=0, \\
M_{y y \lambda} & =-D\left(\frac{\partial^{2} w_{\lambda}}{\partial y^{2}}+v \frac{\partial^{2} w_{\lambda}}{\partial x^{2}}\right)+\lambda \nabla^{2} M_{x x} \\
& =-\frac{\lambda q_{0}}{4 a}[-6 x-2 a+v(6 x-2 a)]+\frac{\lambda q_{0}}{4 a}[-6 x-2 a+v(6 x-2 a)]=0, \\
M_{x y \lambda} & =-D(1-v) \frac{\partial^{2} w_{\lambda}}{\partial x \partial y}+\lambda \nabla^{2} M_{x y}=\frac{3}{2} \frac{\lambda q_{0}}{a}(1-v) y-\frac{3}{2} \frac{\lambda q_{0}}{a}(1-v) y=0 .
\end{aligned}
$$


Indeed, substituting $w=w_{l}+w_{\lambda}$ and the local moments into the nonlocal fundamental relations stated in Sect. 2, it is found that these differential equations as well as the boundary conditions Eq. (9.1) are satisfied. To the best knowledge of the present authors, no results for triangular nonlocal plates have been presented in the literature so far.

\section{Conclusions}

The fundamental relations of the Eringen differential equation-type nonlocal theory of Kirchhoff plates have been summarized in Sect. 2. Corresponding boundary conditions at straight simply supported edges have been discussed in Sect. 3, where a mathematically suitable form has been suggested, see Eq. (11). The validity of the latter boundary condition then has been proofed in Sect. 4. In extension of an analogy originally developed by Marcus [2] for classical local Kirchhoff plates, two Poisson boundary value problems with homogeneous boundary conditions have been presented in Sect. 4, namely for the moment sum and the deflection of the nonlocal Kirchhoff plate, see Eqs. (13), (14), and (19), such that the deflection already satisfies the simple support deflection boundary condition in Eq. (9.1). This representation allows interpreting the moment sum and the nonlocal deflection as the deflection of auxiliary pre-stressed membranes. In this representation, the moment sum turns out to be not affected by the nonlocal effect, see Eq. (18), i.e., it is the same as in the classical local Kirchhoff plate, while the correction of the deflection due to the nonlocal effect is again analogous to an auxiliary pre-stressed membrane, Eq. (22). This immediately leads to the conclusion that the nonlocal correction of the deflection can be directly computed from both, the deflection and the moment sum of the local Kirchhoff plate, Eq. (23). However, up to this point in Sect. 4, only particular solutions of the nonlocal relations stated in Sect. 2 have been presented. In order to show that these solutions are the complete ones for simply supported polygonal nonlocal Kirchhoff plates, particular solutions of Eq. (8) have been presented in Eq. (24) for the Laplace derivatives of the plate moments. Substituting these results into the nonlocal constitutive relations, Eq. (3), it turns out that the moments are the same as the ones delivered by the classical local Kirchhoff theory, i.e., bending and twisting moments are not affected by the nonlocal effect, see Eq. (28). But the moments of the simply supported polygonal local Kirchhoff plate do satisfy the moment boundary condition in Eq. (9.1). It is thus concluded that the solutions presented in Sect. 4 are complete for the case of simply supported nonlocal Kirchhoff plates, since both, the differential equations in Sect. 2 and the boundary conditions in Eq. (9.1), are satisfied. The correction for the deflection due to the nonlocal effect can be computed from the deflection (or, equivalently, from the moment sum) of the local plate according to Eq. (23), and the moments are not affected by the nonlocal effect, Eq. (28). That the local moments vanish for the class of nonlocal plates under consideration can be considered as an important statement. This also holds for the shear forces, due to Eqs. (1.1) and (1.2), and hence also for corner forces and Kirchhoff boundary reaction forces, see, e.g., Ziegler [1] for the latter notions. In Sect. 5, the infinite plate domain, the plate strip, and the equilateral triangular plate are exemplarily discussed, where a comparison to the literature is performed. Also some novel results are presented, such as the Green function for the nonlocal deflection, or the nonlocal deflection of triangular plates. While we have presented analytic formulations in the examples of Sect. 5, it should be noted that numeric results for more complex polygonal plate geometries can be easily obtained by using well-established computational methods for the equally shaped local membrane and plate problems, and applying the above-derived analogies to the corresponding nonlocal plates afterward.

Subsequently, in an Appendix, an analogy between the nonlocal plate deflection and the deflection of a local plate under the action of auxiliary eigenstrain loading is presented.

Acknowledgements Open access funding is provided by Johannes Kepler University Linz. H. Irschik acknowledges support of his work in the framework of the FFG-Comet-K2 "Austrian Center of Competence in Mechatronics (ACCM)," funded by the Federal Government of Austria and the Government of Upper-Austria.

Open Access This article is distributed under the terms of the Creative Commons Attribution 4.0 International License (http:// creativecommons.org/licenses/by/4.0/), which permits unrestricted use, distribution, and reproduction in any medium, provided you give appropriate credit to the original author(s) and the source, provide a link to the Creative Commons license, and indicate if changes were made. 


\section{Appendix: Nonlocal Kirchhoff plate formulated as local Kirchhoff plate with eigenstrain loading in the background}

In this Appendix, we present a re-formulation of the above solutions for nonlocal simply supported Kirchhoff plates. As an alternative to the above presented method of decomposition into membrane-type problems, we utilize the idea of treating the nonlocal plate as a local plate with auxiliary eigenstrain loadings in the background, an idea that extends strategies that were successfully applied before by the group of the late Professor Franz Ziegler to various other fields, e.g., to derive analogies between local plates rigid in shear and local shear-deformable plates, see [13]. We remark that a related eigenstrain-type analogy for the problem of simply supported Bernoulli Euler beams has been presented by Barretta and Marotti de Sciarra [21], see [22] for rods.

In the present case of nonlocal plates, the nonlocal stress resultants are formulated according to a background formulation by means of eigenstrains that might be interpreted as fictitious anisotropic thermal moments $\pi$, see, e.g., [1] for the simpler case of an isotropic thermal expansion,

$$
\pi_{x x}=-\nabla^{2} M_{x x}, \quad \pi_{y y}=-\nabla^{2} M_{y y}, \quad \pi_{x y}=-\nabla^{2} M_{x y} .
$$

Thus, the constitutive relations of the background plate read, see Eqs. (1) and (3),

$$
\begin{gathered}
M_{x x}=-D\left(\frac{\partial^{2} w}{\partial x^{2}}+v \frac{\partial^{2} w}{\partial y^{2}}\right)-\lambda \pi_{x x}, \\
M_{y y}=-D\left(\frac{\partial^{2} w}{\partial y^{2}}+v \frac{\partial^{2} w}{\partial x^{2}}\right)-\lambda \pi_{y y}, \\
M_{x y}=-D(1-v) \frac{\partial^{2} w}{\partial x \partial y}-\lambda \pi_{x y}, \\
Q_{x}=-\frac{\partial}{\partial x}\left(D \nabla^{2} w+\lambda \pi_{x x}\right)-\lambda \frac{\partial}{\partial y} \pi_{x y}, \\
Q_{y}=-\frac{\partial}{\partial y}\left(D \nabla^{2} w+\lambda \pi_{y y}\right)-\lambda \frac{\partial}{\partial x} \pi_{x y} .
\end{gathered}
$$

When inserting these expressions into the equation of motion Eq. (4),

$$
D \nabla^{2} \nabla^{2} w=q-\lambda \frac{\partial^{2}}{\partial x^{2}} \pi_{x x}-\lambda \frac{\partial^{2}}{\partial y^{2}} \pi_{y y}-2 \lambda \frac{\partial^{2}}{\partial x \partial y} \pi_{x y},
$$

it turns out that

$$
\frac{\partial^{2}}{\partial x^{2}} \pi_{x x}+\frac{\partial^{2}}{\partial y^{2}} \pi_{y y}+2 \frac{\partial^{2}}{\partial x \partial y} \pi_{x y}=\nabla^{2} q .
$$

Using the relations of Eq. (5) together with Eq. (18) shows that

$$
\begin{aligned}
& \pi_{x x}=-\frac{\partial^{2} M_{l}}{\partial x^{2}}-v \frac{\partial^{2} M_{l}}{\partial y^{2}}, \\
& \pi_{y y}=-\frac{\partial^{2} M_{l}}{\partial y^{2}}-v \frac{\partial^{2} M_{l}}{\partial x^{2}}, \\
& \pi_{x y}=-(1-v) \frac{\partial^{2} M_{l}}{\partial x \partial y} .
\end{aligned}
$$

As this should be, we finally obtain, see Eq. (19.1):

$$
D \nabla^{2} w=-\left(1-\lambda \nabla^{2}\right) M=-M-\lambda \frac{\pi_{x x}+\pi_{y y}}{1+v}=-M+\nabla^{2} M_{l}=-M-q .
$$


An analogy between the deflection of simply supported nonlocal Kirchhoff plates and the deflection of local Kirchhoff plates with additional eigenstrains has thus been established, see Eq. (A4) for the fourth-order differential equation of the deflection. The fictitious eigenstrains (anisotropic thermal moments) follow according to Eq. (A6) from the local moment sum without an eigenstrain loading. For a simply supported edge, the boundary conditions for Eq. (A4) become:

$$
\Gamma: \quad w=0, \quad D \frac{\partial^{2} w}{\partial n^{2}}=-\lambda \pi_{n n}=\lambda \frac{\partial^{2} M_{l}}{\partial n^{2}}=\lambda \nabla^{2} M_{l}=-\lambda q,
$$

see Eqs. (A2), (A6), and (13), and compare with Eq. (11). Recall that the moments (and shear forces) correspond to the local moments of the plate without an eigenstrain loading. In case of other boundary conditions than those addressed in Eq. (A8), the solutions of Eqs. (A4) and (A8) deliver a particular solution of the nonlocal problem.

\section{References}

1. Ziegler, F.: Technische Mechanik der festen und flüssigen Körper. 3rd edn., Springer, Wien (1998); Mechanics of Solids and Fluids. Corr. 2nd printing, Springer, NewYork (1998)

2. Marcus, H.: Die Theorie elastischer Gewebe und ihre Anwendung auf die Berechnung biegsamer Platten unter besonderer Berücksichtigung der trägerlosen Pilzdecken. Verlag von Julius Springer, Berlin (1924)

3. Eringen, A.C.: Nonlocal Continuum Field Theories. Springer, NewYork (2002)

4. Eringen, A.C.: On differential equations of nonlocal elasticity and solutions of screw dislocation and surface waves. J. Appl. Phys. 54, 4703-10 (1983)

5. Lu, P., Zhang, P.Q., Lee, H.P., Wang, C.M., Reddy, J.N.: Nonlocal elastic plate theories. Proc. R. Soc. Lond. A Math. Phys. Eng. Sci. 463, 3225-3240 (2007)

6. Reddy, J.N.: Nonlocal nonlinear formulations for bending of classical and shear deformation theories of beams and plates. Int. J. Eng. Sci. 48, 1507-1518 (2010)

7. Golmakani, M.E., Rezatalab, J.: Comment on nonlocal third-order sheardeformation plate theory with application to bending and vibration of plates (by R. Aghababaei, J. N. Reddy, Journal of Sound and Vibration 326, 277-289 (2009)). J. Sound Vib. 333, 3831-3835 (2014)

8. Challamel, N., Reddy, J.N.: "Reply to the comments of M.E. Golmakani and J. Rezatalab: "Comment on "Nonlocal thirdorder sheardeformation plate theory with application to bending and vibration of plates" (by R. Aghababaei and J.N.Reddy, Journal of Sound and Vibration 326, 277-289 (2009))“, Journal of Sound and Vibration 333 3831-3835 (2014) “, J. Sound Vib. 333, 5654-5656 (2014)

9. Fernández-Sáez, J., Zaera, R., Loya, J.A., Reddy, J.N.: Bending of Euler-Bernoulli beams using Eringen's integral formulation: a paradox resolved. Int. J. Eng. Sci. 99, 107-116 (2016)

10. Challamel, N., Reddy, J.N., Wang, C.M.: Eringen's stress gradient model for bending of nonlocal beams. J. Eng. Mech. 142, 04016095-1-9 (2016)

11. Romano, G., Barretta, R., Diaco, M., Marotti de Sciarra, F.: Constitutive boundary conditions and paradoxes in nonlocal elastic nanobeams. Int. J. Mech. Sci. 121, 151-156 (2017)

12. Woinowsky-Krieger, S.: Berechnung der ringsum frei aufliegenden gleichseitigen Dreiecksplatte. Ingenieur Arch. 4, 254-262 (1933)

13. Irschik, H., Heuer, R., Ziegler, F.: Statics and dynamics of simply supported polygonal Reissner-Mindlin plates by analogy. Arch. Appl. Mech. 70, 231-244 (2000)

14. Heuer, R., Irschik, H.: A boundary element method for eigenvalue problems of polygonal membranes and plates. Acta Mech. 66, 9-20 (1987)

15. Irschik, H.: Eine Analogie zwischen Lösungen für schubstarre und schubelastische Platten. Z. Angew. Math. Mech. ZAMM 62, T129-T131 (1982)

16. Heuer, R., Irschik, H., Fotiu, P., Ziegler, F.: Nonlinear flexural vibrations of layered plates. Int. J. Solids Struct. 29, 1813-1818 (1992)

17. Irschik, H., Ziegler, F.: Dynamics of linear elastic structures with selfstress: a unified treatment for linear and nonlinear problems. Z. Angew. Math. Mech. ZAMM 68, 199-205 (1988)

18. Irschik, H., Ziegler, F.: Dynamic processes in structural thermo-viscoplasticity. Appl. Mech. Rev. 48, 301-316 (1995)

19. Irschik, H., Ziegler, F.: Application of the Green's function method to thin elastic polygonal plates. Acta Mech. 39, 155-169 (1981)

20. Wang, Q., Liew, K.M.: Application of nonlocal continuum mechanics to static analysis of micro- and nano-structures. Phys. Lett. A 363, 236-242 (2007)

21. Barretta, R., Marotti de Sciarra, F.: Analogies between nonlocal and local Bernoulli-Euler nanobeams. Arch. Appl. Mech. 85, 89-99 (2015)

22. Barretta, R., Marotti de Sciarra, F., Diaco, M.: Small-scale effects in nanorods. Acta Mech. 225, 1945-1953 (2013) 